\title{
EFFECT OF DIAMETER AND BONE DENSITY ON IMPLANT STABILITY IN IMPLANT RETAINED MANDIBULAR OVERDENTURES
}

\author{
M. Shady Nabhan* and Yasmine G. Thabet*
}

\begin{abstract}
Objectives: This study was conducted to evaluate the effect of implant diameter and bone density on the stability of implants in implant retained overdentures.

Materials and methods: Totally, twenty-one patients with entirely edentulous upper and lower arches were randomly assigned into three groups (Group I, II, II). Group (I): patients are exhibiting type I (D1) bone density. Group (II): patients are exhibiting type II (D2) bone density. Group (III): patients are exhibiting type III (D3) bone density. In each patient, two implants with different diameters $(3.5 \mathrm{~mm}$ and $4 \mathrm{~mm}$ )were placed in the intra-foraminal region. Each patient received maxillary conventional complete denture and mandibular implant retained overdenture.Implant stability was measured at implant insertion and after three months.
\end{abstract}

Results: No statistically significant differences were detected regarding implant stability between groups with different bone densities and different diameters. Although there was an increase in implant stability after three months, differences between groups were statistically nonsignificant.

Conclusion: Within the limitations of this study, it was concluded that different implant diameters and bone densities don't have a significant influence on implant stability.

\section{INTRODUCTION}

Loss of teeth leads to resorption of the surrounding alveolar bone thus the outcomes of conventional prosthetic therapy in completely edentulous patients are usually compromised. The drawbacks that occur with conventional complete dentures lead to the development of implant overdentures. Implant overdentures reduce bone resorption, have improved retention and stability and possess a better chewing function, thus improving patients' satisfaction and quality of life. ${ }^{(1,2)}$

Osseointegration is defined as "a direct structural and functional connection between ordered living bone and the surface of a load-carrying implant." (3) It is a prerequisite for successful implant treatment. It is achieved when the healing process occurs without micromotion at the implant-bone interface. ${ }^{(4)}$

* Lecturer of Removable Prosthodontics, Faculty of Dentistry, Ain Shams University 
Implant stability measures the anchorage of implants to the alveolar bone. It has two different stages: primary and secondary stability. It affects the process of osseointegration, the mode of implant loading, and, the success of dental implants. Therefore, improved initial stability and stress-free healing periods were considered as two prerequisites for successful clinical outcomes. ${ }^{(4)}$

Primary stability is defined as "the capacity of the implant to withstand loading in axial, lateral and rotational directions." It is related to the mechanical engagement of the implant with the surrounding bone at insertion, bone quality, and the drilling protocol. Initial implant stability is critical to the success of the implants. Micro-motions with a threshold higher than 50 to $100 \mu \mathrm{m}$ may lead to the formation of fibrous tissue at the bone-implant interface. At the time of implant insertion, assessment of primary stability may act as a guide for selecting the loading protocol: immediate, early or delayed. ${ }^{(4)}$

Secondary stability refers to the increase in implant stability due to regeneration and remodeling of the bone at the bone-implant interface. Adequate primary stability is a prerequisite for achieving secondary stability. ${ }^{(5)}$

Factors affecting implant stability were divided into two categories: factors affecting primary stability and those affecting secondary stability. Factors affecting primary stability include bone morphology (bone quality and quantity), implant geometry (length and diameter of the implant), the surgical technique, and the direction of measurement of implant stability. On the other hand, factors that affect secondary stability include primary stability, bone remodeling, and implant surface characteristics. ${ }^{(6-8)}$

The amount and density of the available bone in the edentulous arches are the primary determining factors in predicting implant success. The osseointegration process is affected by different levels of bone cellularity and vascularity at the site of implantation. A healthy bone bed, as well as minimal surgical trauma, contribute to the bone healing response. ${ }^{(9)}$

Misch (10) classified the bone according to its density, based on macroscopic characteristics, into four divisions. They range from the densest D1 (Dense cortical bone) to the least dense D4 (fine trabecular bone). D1is usually found in the anterior part of the mandible, D2 (Dense to the thick cortical bone and coarse trabecular bone) is usually found in the mandible and the anterior part of the maxilla. D3 (Thinner porous cortical bone and fine trabecular bone) is found in the maxilla and the posterior part of the mandible while D4 might be found in the posterior part of the maxilla. A very soft bone with incomplete mineralization could be classified as D5, which is usually an immature bone. The bone density is usually determined by the location, radiographic evaluation or tactile sense during surgery.

Several studies revealed a higher survival rate for implants placed in the mandible, particularly in the anterior region. On the other hand, a lower survival rate of the implants placed in the maxilla was reported. The lower success rate in low-density bone may be due to the reduced biomechanical anchorage of the implants in trabecular bone. Thus, the survival rates of the implants are related to the bone conditions surrounding the implants. Therefore, the volume and quality of the bone are important factors for the success of dental implants. ${ }^{(11,12,13)}$

Studies were done to determine factors improving implant stability. The osteotome technique for bone condensation was developed to achieve improved primary stability. The objective of this procedure is to compress the bone laterally and axially to create a precisely formed implant site without any bone loss. Moreover, it was reported that an implant of increased width might increase primary stability. It was found that implant diameter has a better effect than implant length in the distribution of loads to the 
surrounding bone. The use of wider implants allows the engagement of a maximal amount of bone. ${ }^{(14,15)}$ It was proved that more bone contact provides increased initial stability and resistance to stresses. ${ }^{(16)}$ Thus this study was conducted to evaluate the effect of different implant diameters placed in different bone densities on the stability of implants in implant retained mandibular overdentures.

\section{MATERIALS AND METHODS}

Eighteen patients were selected from the outpatient clinic of the Prosthodontic Department, Faculty of Dentistry, Ain Shams University to participate in this study. Inclusion criteria: completely edentulous arches with residual ridges of adequate height and width covered by firm, dense fibrous mucoperiosteum, free of any pathological signs, bony undercuts or neoplastic lesions and with normal maxillo-mandibular Angle's class I skeletal relationship. Exclusion criteria: patients with inadequate inter-arch space, neuromuscular disorders, parafunctional habits, systemic diseases that affect healing and smokers.

All participants were informed in details about the nature of the investigation, the purpose of the study and signed an informed consent form. Computed Tomographic x-ray (CT) was made for all patients with the radiographic stent to determine the bone density at the proposed implant sites (with the aid of the imaged guttapercha). The average CT values of the bone surrounding the simulated implants were calculated by the software through calculating the Hounsfield unit (HU).(Fig.1)

All the selected patients were rehabilitated with mucosa supported maxillary complete dentures and implant retained mandibular overdentures which were constructed following the basic principles. Two implants with different diameters were installed at the inter-foraminal region. Patients were classified into three groups according to the bone density at the site of implant placement.

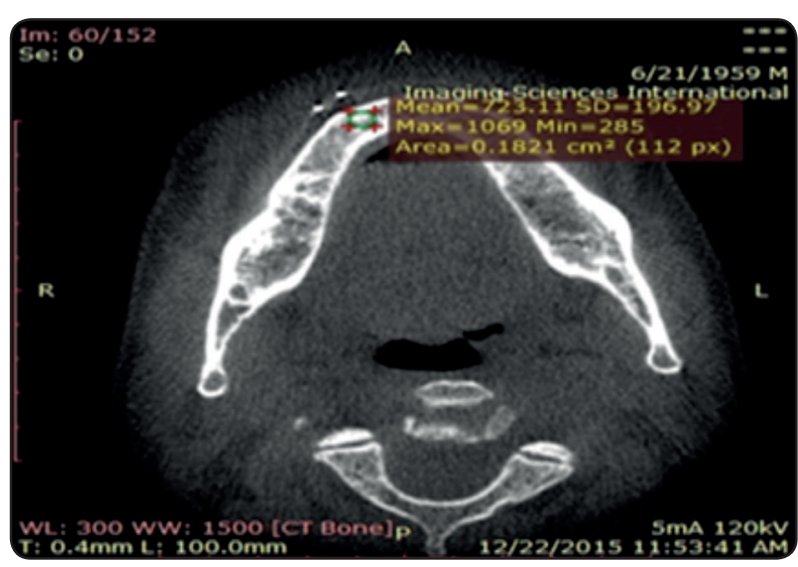

Fig. (1) C.T. used to calculate Housefield unit at the implant site.

Group I: Six patients exhibiting type I (D1) bone density (HU ranged from 1255-1350).

Group II: Six patients exhibiting type II (D2) bone density (HU) ranged from (750-900).

Group III: Six patients exhibiting type III (D3) bone density (HU) ranged from (568-720).

The radiographic stent was modified to act as a surgical stent. Holes were made in the position of the proposed implants to guide their insertion in the canine region. Two tapered threaded (two pieces) implants (INNO Internal implant, Cowellmedi Co., Ltd.) 3.5 and $4 \mathrm{~mm}$ in diameter and $10 \mathrm{~mm}$ in length were used.

The patient was anesthetized, and a mid-crestal incision was made using no. 15 blade which extended $(1-1.5 \mathrm{~cm})$, beyond the proposed area for the implants, mesially and distally. The mucoperiosteal flap was elevated, and the bone site was marked, using the surgical stent, and prepared following the drill sequence recommended by the implant manufacturer. The implants were manually threaded in the osteotomy site using the implant mount in a clockwise direction submerging the apical third; then the ratchet wrench was used to complete the implant insertion until the cervical part was nearly flushed with the crestal bone. (Fig.2) 


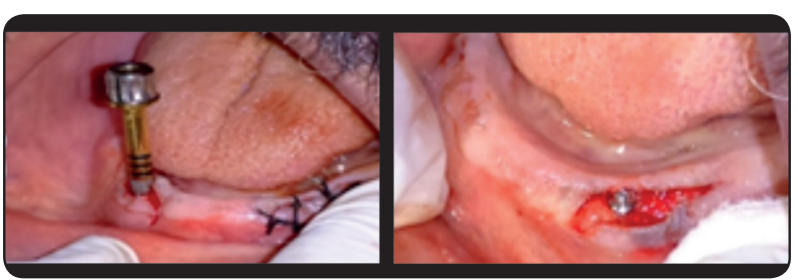

Fig. (2) Insertion of the implants in the canine region.

\section{Measurement of the implant stability}

After the implants were installed, the initial implant stability was measured using the 'Osstell' ISQ device. This device measures the implant stability through a resonance frequency analyzer and a transducer (magnetic peg). The smart peg (transducer) is a metallic rod with a magnet on top that is screwed onto an implant or an abutment by a smart peg holder. The magnet is activated by a magnetic pulse from the wireless probe. After excitation, the peg vibrates freely, and the magnet induces an electric voltage in the probe coil. This voltage is the measurement signal sampled by the resonance frequency analyzer (RFA). The results of the resonance frequency analyzer are expressed as an implant stability quotient (ISQ) on a scale from 1 to 100 , which represents a standardized unit of stability.

The smart pegs (transducers) were selected according to the reference guide available from OSSTELL, as each implant system has its compatible smart peg. The magnetic pegs were fully screwed to the implants using the peg holder, and the 'electric probe' was approximated on top of the magnetic peg, till a reading was obtained, which indicates implant stability quotient (ISQ). After all, readings were recorded for all implants, the pegs were removed using the peg holder. Then the cover screws of the implants were screwed in position. After that, the flap was replaced and carefully adapted over the cover screw and sutured.

The patients were recalled after three months for measuring the implant stability before loading.
The surgical stent was used to mark the implant site, and a lancet was used to expose the implants, then the cover screws were removed from the patient's mouth. The smart pegs were screwed again to the implants and recordings were made. (Fig. 3)

Ball abutments with suitable heights were selected and seated with the ball wrench. The metal housings with the O-rings were placed and accurately fitted to the metal balls of the abutments. Relief of the fitting surface of the lower denture was done opposite to the ball abutments of the implants. An elastomeric shim (spacer) was placed at the cervical half of the balls to block out undercuts, and the self-cured acrylic resin was used for denture pick up. The denture was then returned to patient's mouth to ensure complete seating, and any necessary adjustments were carried out to eliminate occlusal interferences.

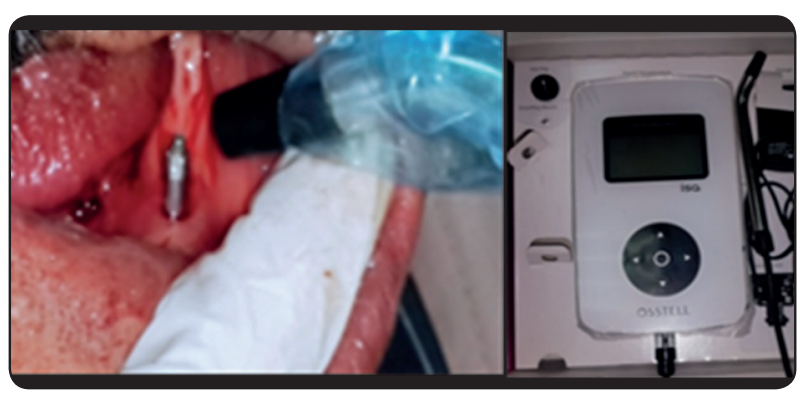

Fig. (3) Measurement of implant stability at loading.

\section{Statistical analysis}

Data were explored for normality by checking the data distribution, calculating the mean and median values and using Kolmogorov-Smirnov and Shapiro-Wilk tests. Data showed normal distribution.

Data were presented by mean and standard deviation (SD) values. Mixed ANOVA was used to assess the effect of implant diameter and bone density over implant stability.The significance level was set at $\mathrm{P} \leq 0.05$. 
Statistical analysis was performed with IBM ${ }^{\circledR}$ SPSS ${ }^{\circledR}$ Statistics Version 20 for Windows.

\section{RESULTS}

\section{Comparison between the three groups at insertion:}

TABLE (1): The mean, standard deviation (SD) values and $p$ values of stability of narrow and wide implants in D1, D2, and D3 bone at implant insertion.

\begin{tabular}{|c|c|c|c|c|c|}
\hline \multirow[b]{2}{*}{ Insertion } & \multicolumn{2}{|c|}{ Narrow } & \multicolumn{2}{|c|}{ Wide } & \multirow[b]{2}{*}{$\begin{array}{c}\mathrm{P} \\
\text { value }\end{array}$} \\
\hline & Mean & $\begin{array}{c}\text { Std. } \\
\text { Deviation }\end{array}$ & Mean & $\begin{array}{c}\text { Std. } \\
\text { Deviation }\end{array}$ & \\
\hline D1 & 67.67 & 5.96 & 68.83 & 4.92 & 0.67 \\
\hline D2 & 64.83 & 3.06 & 68.5 & 2.95 & 0.186 \\
\hline D3 & 67.5 & 3.95 & 68.83 & 6.24 & 0.626 \\
\hline$P$ value & \multicolumn{2}{|c|}{0.25} & \multicolumn{2}{|c|}{0.925} & \\
\hline
\end{tabular}

At implant insertion, wide diameter implants showed higher implant stability (ISQ value) compared to narrow diameter implants, however, ANOVA test showed no statistically significant difference between the studied groups.

Regarding narrow implant diameter, D2 bone density showed the lowest value compared to D1 and D3, however, differences were statistically non-significant. Moreoever, different bone densities showed statistically non-significant difference with wide diameter implants.

TABLE (2): The mean, standard deviation (SD) values and $p$ values of stability of narrow and wide implants in D1, D2, and D3 bone at implant loading.

\begin{tabular}{|c|c|c|c|c|c|}
\hline \multirow[t]{2}{*}{ Loading } & \multicolumn{2}{|c|}{ Narrow } & \multicolumn{2}{|c|}{ Wide } & \multirow[t]{2}{*}{$P$ value } \\
\hline & Mean & $\begin{array}{c}\text { Std. } \\
\text { Deviation }\end{array}$ & Mean & $\begin{array}{c}\text { Std. } \\
\text { Deviation }\end{array}$ & \\
\hline D1 & 74.17 & 6.91 & 74.17 & 5.03 & 1 \\
\hline D2 & 69.33 & 3.1 & 75.33 & 3.08 & 0.51 \\
\hline D3 & 72.83 & 4.62 & 73.5 & 4.64 & 0.809 \\
\hline $\mathrm{P}$ value & \multicolumn{2}{|c|}{0.662} & \multicolumn{2}{|c|}{0.178} & \\
\hline
\end{tabular}

At implant loading, there were similar results as there was no significant difference between narrow and wide diameter implants and there was no significant difference between different bone densities.

Although implant stability increased at the time of loading compared to stability at all subgroups however difference was statistically nonsignificant. Fig(4)

TABLE (3): The mean, standard deviation (SD) values and $p$ values of stability of narrow and wide implants in D1, D2, and D3 bone at implant insertion and at loading.

\begin{tabular}{|c|c|c|c|c|c|c|}
\hline \multirow{2}{*}{} & & \multicolumn{2}{|c|}{ Insertion } & \multicolumn{2}{c|}{ Loading } & \multirow{2}{*}{ P value } \\
\cline { 2 - 7 } & & Mean & Std. Deviation & Mean & Std. Deviation & \\
\hline \multirow{3}{*}{ Narrow } & D1 & 67.67 & 5.96 & 74.17 & 6.91 & 0.084 \\
\cline { 2 - 7 } & D2 & 64.83 & 3.06 & 69.33 & 3.1 & 0.976 \\
\cline { 2 - 7 } & D3 & 67.5 & 3.95 & 72.83 & 4.62 & 0.772 \\
\hline \multirow{3}{*}{ Wide } & D1 & 68.83 & 4.92 & 74.17 & 5.03 & 0.893 \\
\cline { 2 - 8 } & D2 & 68.5 & 2.95 & 75.33 & 3.08 & 0.555 \\
\cline { 2 - 7 } & D3 & 68.83 & 6.24 & 73.5 & 4.64 & 0.92 \\
\hline
\end{tabular}




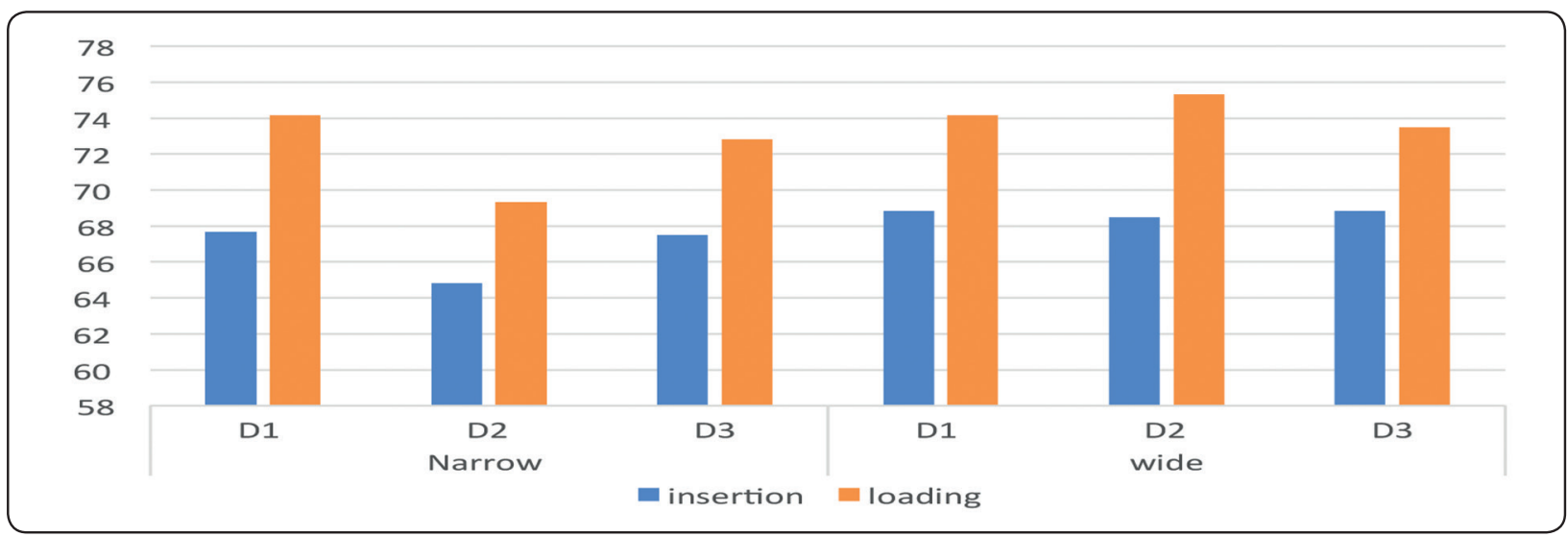

Fig. (4) Stability values of narrow and wide implants in D1, D2, and D3 bone at insertion and loading.

\section{DISCUSSION}

This study was conducted to evaluate the effect of using implants of different diameters in different bone densities on implant stability. Primary stability of implants mainly depends on bone-to-implant contact area. The bone quality and implant length and diameter have been assumed to be influential on the bone-to-implant contact and consequently on implant primary stability. ${ }^{(17)}$

It has been reported that implants with better initial stability have higher secondary stability and require reduced healing periods than those with lower initial stability. The measurement of secondary stability will indicate the success of healing. ${ }^{(18)}$

Studies revealed that $\mathrm{CT}$ is an objective and reliable tool for the assessment of the bone density of patients requiring implant therapy. Bone density at the implant sites is determined by computer tomographic data using implant planning software which can calculate Hounsfield units (HU). The Hounsfield unit (HU) is a quantitative assessment of bone density measured by its ability to attenuate an X-ray beam. ${ }^{(19,20,21)}$

The resonance frequency analysis provides an objective measurement of implant stability which is measured by an implant stability quotient (ISQ) given by the Osstell. The implant stability quotient (ISQ) ranges from 1 to100, where values less than 45 indicate failure of the implant, whereas values of about 60 to 70 indicate implant success. ${ }^{(22)}$

According to Misch, the capacity of bone to withstand physiologic loads differs according to the bone quality, with the highest strength for D1 bone and the lowest strength with D4 bone quality. ${ }^{(10)}$ This coincides with the results of this study where there was a relation between primary stability and bone density. Although the primary stability was higher in high bone quality (D1) compared to lower bone quality (D2, D3) yet this difference was statistically insignificant. This difference may be attributed to the functional surface area between the implant and the bone, in addition to the higher mechanical tolerance of D1 bone to functional load.

A study stated that the presence of cortical bone, which is 10 to 200 times more rigid than cancellous bone, can be the cause of high primary stability in high bone quality. ${ }^{(23)}$ Furthermore, another study found that implant stability values increase predominantly in loose trabecular bone during osseointegration, while in compact bone, the implants had higher stability values due to better primary me- 
chanical stability. Thus cortical thickness is important for primary implant stability. ${ }^{(24)}$

Implants seem be more stable in cortical bone compared to trabecular bone. ${ }^{(25)}$ However, a study suggested that following a 4-6 months healing period, implants placed in different bone densities approach towards a similar level of secondary stability. ${ }^{(26)}$ It seems that implants inserted in lowdensity bone "catch up" over time with those placed in bone of medium and high density. (27) These findings support the results of this study where there was no significant difference between narrow and wide diameter implants in different bone densities at the time of loading.

A study suggested using wider diameter implants in low-density bone to gain higher primary stability. ${ }^{(28)}$ It has been revealed that incorporating wider diameter implants increases the bone-implant contact not only to the crestal cortical layer but also at the lateral cortical walls. ${ }^{(27)}$ It has been reported that surface-modified implants maintain implant stability during the first three months of healing in contrast to the machined surface implants. Although surface texturing of implants does not directly contribute to initial implant stability, it may reduce the risk of loss of stability and consequently facilitate wound healing (secondary osseointegration). ${ }^{(29)}$

A direct relationship was observed between implant stability and implant diameter, however not between implant stability and implant length. ${ }^{(30)}$ Although wider diameter implants showed higher ISQ values both at insertion and loading, however, these differences were statistically insignificant.

\section{CONCLUSION}

Within the limitations of this study, it was concluded that different implant diameters and bone densities don't have a significant influence on implant stability.

\section{REFERENCES}

1- Rashid F., Awad M A,.Thomason J M., Piovano A., Spielberg GP., Scilingo E.,Mojon P., Muller F., Spielberg M., Heydecke G., Stoker G.,Wismeijer D., Allen F., Feine J.: The effectiveness of 2-implant overdentures - a pragmatic international multicentre study, J. Oral Rehabil., 2011, 38:p. 176-184.

2- Assuncao W. G., Barao V. A. R., Delben J. A., Gomes E. A. \& Tabata L. F.: A comparison of patient satisfaction between treatment with conventional complete dentures and overdentures in the elderly: a literature review, Gerodontology, 2010,27: p 154-162

3- Brånemark R., Rydevik B. \& Myers R.: Osseointegration in skeletal reconstruction and rehabilitation, J. Rehabil. Res. Dev., 2001,38:p 1-4

4- Turkyilmaz I., Sennerby L., McGlumphy E. A. \& Tözüm T. F.: Biomechanical aspects of primary implant stability: A human cadaver study, Clin. Implant Dent. Relat. Res., 2009, 11: p 113-119

5- Jung U., Kim S., Lee In.: Secondary stability of micro thickness hydroxyapatite-coated dental implants installed without primary stability in dogs, Clin. Oral Implants Res., 2014,2: p 1169-1174

6- Atsumi M., Park S.H. \& Wang H.L.: Methods used to assess implant stability: current status, Int. J. Oral Maxillofac. Implants, 2007, 22: p 743-54

7- Eliasa C.N., Rocha F.A., Nascimento A. L. \& Coelho P. G.: Influence of implant shape, surface morphology, surgical technique and bone quality on the primary stability of dental implants, J. Mech. Behav. Biomed. Mater., 2012, 16: p 169-180

8- Meredith N.: Assessment of implant stability as a prognostic determinant, Int. J. Prosthodont., 1998, 11: p 491-501

9- Zarb G.A. \& Alberktsson T.: Criteria for determining clinical success with osseointegrated dental implants, Cah. Prothese.,1990,71: p 19-26

10- Misch C.E. (2008), "Dental implants Prosthetics", $3^{\text {rd }}$ ed., Mosby Inc.,St Louis, Canada

11- Fuh L. J., Huan H. L., Chen C. S., Fu K. L., Shen Y. W., Hsu J. T.: Variations in bone density at dental implant sites in different regions of the jawbone, J. Oral Rehabil., 2010, 37: p 346-351

12- Kacer C. M., Dyer J. D. \& Kraut R. A.: Immediate loading of dental implants in the anterior and posterior mandible: A retrospective study of 120 cases, J. Oral Maxillofac. Surg.,2010, 68: p 2861-2867 
13- Ko Y., Chun H., Heng Li.: Variations in crestal cortical bone thickness at dental implant sites in different regions of the jawbone, Clin. Implant Dent. Relat. Res., 2017, 19: p 440-446

14- Summer R.B., Mawr B.: The osteotome technique: part 2 -the ridge expansion osteotomy procedure, Compend Contin. Educ. Dent., 1994,15: p 422-434.

15- Khon D.H.: Overview of factors important in implant design, J. Oral Implantol.,1992,18(3): p 204-219

16- Misch C.E.: Implant design considerations for the posterior regions of the mouth, Implant Dent.,1999, 8(4): p 376-386.

17- Doundoulakis J. H., Eckert S. E., Lindquist C. C. \& Jeffcoat M. K.: The implant-supported overdenture as an alternative to the complete mandibular denture, J. Am. Dent. Assoc., 2003, 134: p 1455-1458

18- Huang H.M., Chiu C.L., Yeh L.C., Lin C.T., Lin L.H., Lee S.Y.: Early detection of implant healing process using resonance frequency analysis, Clin. Oral Implants Res., 2003, 14: p 437- 43 .

19- Bennani V. \& Serre D.: Radiographic stent for a quick and precise bone height analysis, J. Prosthet. Dent.,2000, 83: $480-481$

20- Mccord J. F. \& Grant A. A.: Technical aspects of complete denture construction, Br. Dent. J.,2000, 189: p 71-74

21- Farré-Pagès N., Augé-Castro M., Alaejos-Algarra F.: Relation between bone density and primary implant stability, Med. Oral Patol. Oral Cir. Bucal, 2011,16(1): p 62-7

22- Sennerby L., Meredith N. \& Petersson A.: In Implant Den- tistry Research Guide: Basic, Translational and Clinical Research, 2012,p 513-538

23- Resnik RR.: "Dental Implant Prosthetics", Dent. Implant Prosthetics, 2015, 2: p 126-158

24- Sim C.P.C. and Lang NP.: Factors influencing resonance frequency analysis (RFA) assessed by Osstell mentor during implant tissue integration. I. Instrument positioning, bone structure, implant length, Clinical Oral Implants Research, 2010, 21: p 598-604

25- Sennerby L., Thomsen P., Ericson L.E.: Early bone tissue responses to titanium implants inserted in rabbit cortical bone (I). Light microscopic observations, J. Mater Sci Mater Med, 1993, 4: p 240-50.

26- Sennerby L. and Meredith N.: Diagnostic de la stabilitéd'un implant par l'analyse de sa fréquence de résonance. Implant, 1999, 5: p 93-100

27- Friberg B.: Surgical approach and implant selection (Brånemark system) in bone of various densities, Appl. Osseointegrat. Res., 2002, 3(1): p 9-16

28- Friberg B.: On bone quality and implant stability measurements (thesis), Göteborg: University of Göteborg; 1999.

29- Glauser R., Portmann M., Ruhstaller P. et al.: Stability measurements of immediately loaded machined and oxidized implants in the posterior maxilla, Appl. Osseointegr. Res., 2001, 2(1): p 27-9

30- Vidyasagar L. Salms G., Apse P., Teibe U.: Dental Implant Stability at Stage I and II Surgery as Measured Using Resonance Frequency Analysis, Stomatologija, Baltic Dental and Maxillofacial J., 2004, 6: p 67-72 